\title{
Seasonality of Clostridium difficile infections in Southern Germany
}

\author{
M. REIL ${ }^{1}$, M. P. M. HENSGENS ${ }^{2}$, E. J. KUIJPER ${ }^{2}$, T. JAKOBIAK ${ }^{1}$, H. GRUBER $^{1}$, \\ M. KIST ${ }^{3}$ AND S. BORGMANN ${ }^{1,4 *}$ \\ ${ }^{1}$ Synlab Medical Care Service, Medical Care Center Weiden, Weiden, Germany \\ ${ }^{2}$ Department of Medical Microbiology, Leiden University Medical Center, Leiden, The Netherlands \\ ${ }^{3}$ Department of Microbiology and Hygiene, University Hospital Freiburg, Freiburg, Germany \\ ${ }^{4}$ Department of Infectious Diseases and Infection Control, Klinikum Ingolstadt, Ingolstadt, Germany
}

(Accepted 17 November 2011; first published online 8 December 2011)

\section{SUMMARY}

Between 2000 and 2009, the total number of patients with Clostridium difficile infections increased considerably in Southeastern Germany. A clear seasonality was observed with a higher number of affected patients occurring in the winter months (January-March). Moxifloxacin and erythromycin-resistant $C$. difficile PCR ribotypes 001 (72\%) and $027(4 \cdot 6 \%)$ were the most commonly isolated strains.

Key words: Antibiotic resistance, Clostridium difficile, typing.

\section{INTRODUCTION}

In the last decade increasing numbers of Clostridium difficile infections (CDI) have been reported in Northern America and Europe [1]. Similarly in Germany an increased number of CDI affected inpatients was observed between 2000 and 2004 [2]. A previous study reported that the number of $C$. difficile toxin (Tcd)-positive patients (in- and outpatients) in Southeastern Germany markedly increased between 2000 and 2006 [3]. That study described ribotype 001 to be the most common strain, while the recently emerging ribotype 078 [4] was isolated sporadically. Ribotype 027 was not found [3]. Severe CDI and the identification of CDI ribotype 027 are notifiable in Germany [5]. Severe clinical disease was associated not only with polymerase chain reaction (PCR) ribotype 027 , but also with types 017 and 001 [6-8]. As

\footnotetext{
* Author for correspondence: Dr S. Borgmann, Department of Infectious Diseases and Infection Control, Klinikum Ingolstadt, Krumenauerstr. 25, D-85049 Ingolstadt, Germany. (Email: Synlab@gmx.de)
}

reported in other countries, $C$. difficile strains belonging to PCR ribotype 027 have reduced susceptibilities to newer generation fluoroquinolones [7]. A disadvantage of the current German surveillance programme is the fact that only selected cases of CDI, those with severe clinical disease, are reported to the health authorities. To address this gap, we prospectively analysed all patients with CDI identified by a laboratory in Southeastern Germany in 2009. This laboratory provides medical services to a high proportion of regional hospitals and ambulatory physicians [3]. Results of this study provided a better representative overview of the epidemiology and antimicrobial resistance of $C$. difficile isolated in a rural region in Southeastern Germany compared to standard surveillance practices.

\section{PATIENTS AND METHODS}

\section{Laboratory and hospital settings}

The Synlab Medical Care Service Centre Weiden, located in Northern Bavaria (Southeastern Germany), 
analyses laboratory samples from about 40 hospitals and over 2000 physicians serving outpatients and is a source for the epidemiological data as described previously $[3,9]$. To calculate the number of Tcd-positive patients per period (months/3 months/year) data from the laboratory data system (MCS, Germany) were used. Patients with multiple positive tests in one time period were included only once. All data were loaded into the Hybase program (Cymed AG, Germany) (http://www.hyplan.de/download_hy.php). Hybase is a computer program used for infection surveillance in a given institution, for instance to summarize the number of defined pathogens, e.g. notifiable bacteria. Due to limited information regarding hospital and community onset of CDI, in- and outpatients were classified based on the facility submitting samples for Tcd analysis to the laboratory (ambulatory physicians $=$ outpatients, hospitals $=$ inpatients $)$.

\section{Tcd analysis and culture}

All stool samples sent to our laboratory in 2009 requesting CDI testing were included in this study. CDI testing was performed by ELISA for Tcd A and B (R-Biopharm AG, Germany). Stool samples with a positive Tcd test were cultured for $C$. difficile as previously described [3]. Identification of $C$. difficile was performed by rapid ID 32 A system (identification system for anaerobes; bioMérieux, Germany) and by matrix-assisted laser desorption/ionization time-offlight mass spectrometry (MALDI-TOF MS) microflex LT system (Bruker Daltonik GmbH, Germany).

\section{PCR ribotyping and antibiotic susceptibility testing}

To get a representative overview of the $C$. difficile situation in our region, using PCR we ribotyped all isolates cultured in 2009 according to the protocol described by Bidet et al. [10]. PCR ribotype profiles were compared to banding patterns of $25 \mathrm{C}$. difficile reference strains (Cardiff, ECDC collection). C. difficile isolates which did not match any of the reference strains were sent to the German reference laboratory for gastrointestinal infections (University of Freiburg, Freiburg, Germany) or to Leiden University Medical Center (LUMC, Leiden, The Netherlands) for further typing.

Antimicrobial susceptibility testing for moxifloxacin, erythromycin and ciprofloxacin was conducted by E-test (bioMérieux, Germany) analysis. A total of 34 isolates were examined. Ten isolates of ribotype 001,14 isolates of 027 and ten isolates of sporadic strains were randomly harvested for antibiotic susceptibility testing. Cut-off values for antimicrobial susceptibility were assessed according to Barbut et al. [11] (erythromycin $\geqslant 4 \mathrm{mg} / 1$, moxifloxacin $\geqslant 4 \mathrm{mg} / \mathrm{l})$. Ciprofloxacin resistance was defined at a minimal inhibitory concentration (MIC) $\geqslant 2 \mu \mathrm{g} / \mathrm{ml}$.

\section{Statistics}

The total number of patients tested and the number of positive patients with a positive Tcd test were used to calculate the percentage of positive patients. An autoregressive model was applied to the data to test whether the total number of patients tested for CDI, the total number of positive patients or the percentage of patients positive for CDI followed a seasonal pattern. In this model, we studied the four seasons, while taking into account a linear increase in the total number of isolates in time (Fig. 1). Because the number of patients in a season can be influenced by the number of patients in the previous season(s), we applied a first-order autoregression, which considers the result of the previous season. Taking into account more than one previous season did not improve our model.

Differences in ribotype frequencies between in- and outpatients were evaluated by $\chi^{2}$ calculation. $P$ values were corrected for multiple comparisons (total number of identified ribotypes) by the Bonferroni procedure.

\section{RESULTS}

Figure 1 depicts the annual number of CDI patients identified from 2000 to 2009. During this period the total number of patients examined for Tcd per year increased 5.8-fold from 1453 to 8449 (Fig. 1a) (regression coefficient: +49 requests per 3 months). This increase was predominantly caused by a higher number of laboratory requests for inpatients, but requests for outpatients also rose. The number of Tcd-positive patients increased $10 \cdot 1$-fold from 95 to 899 (Fig. 1b) (regression coefficient: +6 positive patients per 3 months); however, the percentage of positive patients varied between $5 \%$ and $19 \%$ and did not increase consistently in time (Fig. 1c). The total number of tests requested and the number of positive Tcd stool samples were more frequently found within the first months of each year, indicating possible 

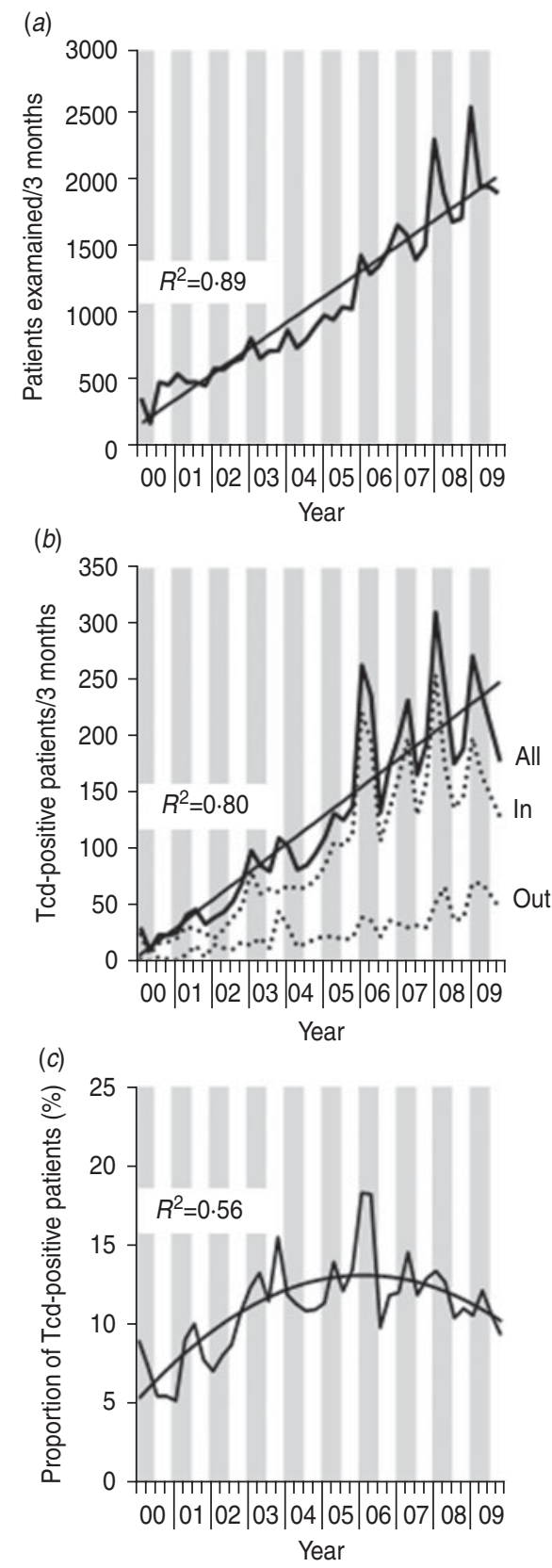

Fig. 1. C. difficile infections in Southern Germany (Bavaria) per 3 months between 2000 and 2009. Stool samples were examined at the Synlab Medical Care Center Weiden. Grey vertical bars represent winter (January-March) and spring (May-June) quarters; white vertical bars indicate summer (July-September) and autumn (October-December) quarters. (a) Number of patients examined for $C$. difficile toxin (Tcd) carriage. (b) Number of Tcd-positive patients. All = number of Tcd-positive inpatients (In) and outpatients (Out). (c) Proportion of Tcd-positive patients.

seasonal influence. To investigate the presence of a seasonal effect, we applied an autoregression model. After adjusting for the strong linear component

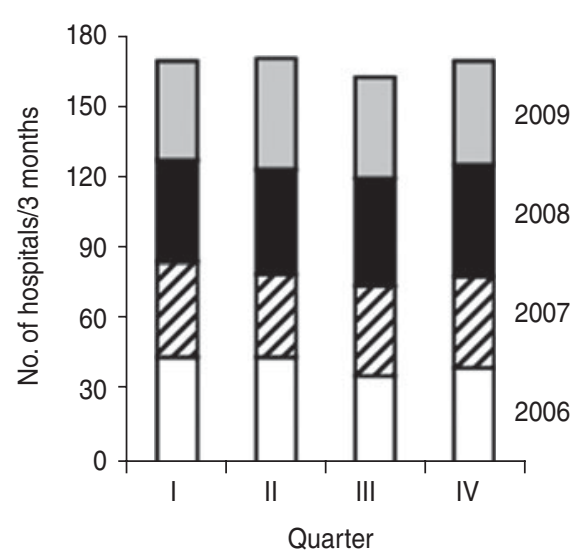

Fig. 2. Absolute number of hospitals submitting stool samples to the Synlab Medical Care Center Weiden per 3-month period (quarters) between 2006 and 2009. Most hospitals are general hospitals although a few teaching hospitals are also included (I, January-March; II, AprilMay; III, June-Augist; IV, September-December).

$(P<0 \cdot 01)$ of the total number of isolates, the number of isolates tested in the months January, February and March $(P<0 \cdot 01)$ appeared to be significantly more than the number of isolates tested in the rest of the year. The same phenomenon was found for the absolute number of positive patients. The relative number of positive isolates was not related to certain months (all $P>0 \cdot 20$ ), using the same analysis. Data about hospitals submitting stool samples to our laboratory was available from 2006 to 2009 . In this period the number of hospitals did not substantially change over time (Fig. 2). Therefore, the number of stool examinations increased because hospitals submitted more stool samples.

PCR ribotyping was conducted on 587 isolates; 488 $(83.2 \%)$ came from inpatients and $99(16 \cdot 8 \%)$ from outpatients. Out of 587 typed strains, $423(72 \cdot 1 \%)$ were PCR ribotype 001, $27(4 \cdot 6 \%)$ type 027,17 $(2.9 \%)$ type $077,14(2 \cdot 4 \%)$ type $023,11(1.9 \%)$ type $126,10(1 \cdot 7 \%)$ type 78 , and $9(1.5 \%)$ ribotype 015 . Rarely found PCR ribotypes were 002 and 095 $(n=8), 012$ and $056(n=6), 029$ and $046(n=5), 014$, 081 , and $149(n=4), 003$ and $005(n=3), 017,045$, 054, 070, 087, 106, and $150(n=2), 020,031,057,097$, 117 , and $131(n=1)$, respectively.

A difference in the distribution of various PCR ribotypes in inpatients and outpatients was found only for PCR ribotype 001 . The proportion of ribotype 001 was significantly higher in inpatients $(75.0 \%)$ compared to outpatients $(57 \cdot 6 \%)(P=0 \cdot 01)$. The distribution of various PCR ribotypes across time did not 
Table 1. Antibiotic resistance pattern of $\mathrm{C}$. difficile strains isolated from patients in Northern Bavaria in 2009

\begin{tabular}{lllll}
\hline \hline & \multicolumn{2}{l}{ Minimal inhibitory concentration $(\mu \mathrm{g} / \mathrm{ml})$} & \\
\cline { 2 - 4 } Ribotype & Moxifloxacin & Ciprofloxacin & Erythromycin & No. of isolates \\
\hline 001 & $>32$ & $>32$ & $>256$ & 8 \\
& $0 \cdot 38$ & 12 & $0 \cdot 25$ & 1 \\
027 & $0 \cdot 25$ & 1 & 8 & 1 \\
& $>32$ & $>32$ & $>256$ & 12 \\
012 & $>32$ & 12 & $>256$ & 1 \\
015 & $0 \cdot 19$ & 4 & $0 \cdot 125$ & 1 \\
023 & $0 \cdot 38$ & $16-24$ & $<\cdot 0 \cdot 016$ & 1 \\
029 & 4 & $>32$ & $0 \cdot 094$ & 1 \\
046 & $0 \cdot 38$ & 24 & $0 \cdot 25$ & 1 \\
057 & $>32$ & $>32$ & $>256$ & 1 \\
077 & $1 \cdot 5$ & $>32$ & $0 \cdot 19$ & 1 \\
087 & $0 \cdot 19$ & $>32$ & $>256$ & 1 \\
126 & $>32$ & 4 & $0 \cdot 125$ & 1 \\
126 & $0 \cdot 25$ & $>32$ & $>256$ & 1 \\
\hline \hline
\end{tabular}

reveal a seasonal correlation. Of the 34 strains tested for antimicrobial susceptibility, only one isolate was susceptible to ciprofloxacin. Resistance to erythromycin and moxifloxacin was common in $C$. difficile types 001 and 027 but rare in the other ribotypes (Table 1).

In order to study the coincidence of CDI and norovirus diarrhoea the number of norovirus notifications from Bavaria were requested from the Robert-KochInstitute (RKI). From 2000 to 2006 only a few cases of norovirus infection had been reported. However, from 2007 to 2009 the number of notified norovirus infections coincided with increased numbers of CDI (Fig. 3).

\section{DISCUSSION}

Since 2000, an increase of patients with CDI was observed in a rural region in Southeastern Germany. The number of patients tested for CDI also showed an increase. Therefore, the percentage of positive patients increased only from $5-10 \%$ to $10-15 \%$. The increase in the number of patients tested for CDI and the number of CDI-positive patients presenting during 2000 and 2009 followed a seasonal pattern, with the largest numbers present in the winter months. Most commonly ribotype 001 was found, especially in inpatients.

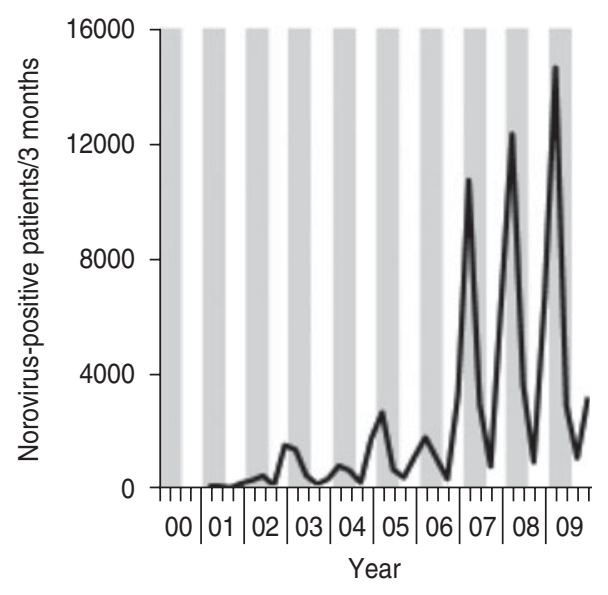

Fig. 3. Number of notified norovirus infections in Bavaria (Southeastern Germany) between 2000 and 2009. Grey vertical bars represent winter (January-March) and spring (May-June) quarters; white vertical bars indicate summer (July-September) and autumn (October-December) quarters. Data were obtained from the SurfStat database of the German Robert-Koch-Institute (http://www3.rki.de/ SurvStat/QueryForm.aspx).

The general increase in incidence of CDI has been observed previously [1]. This can partly be due to an increased awareness of physicians, stimulated by publication of national and European guidelines and the mandatory surveillance for (severe) CDI.

Most $C$. difficile isolates showed high MICs to ciprofloxacin. In our region, ciprofloxacin prescription 
numbers markedly increased from 2000 to 2006 with an apparent correlation with the number of Tcdpositive patients [12]. Excluding the first half of 2006, a linear increase of CDI numbers is seen within this period (Fig. 1b). Interestingly, when summing up prescription numbers of ciprofloxacin and cotrimoxazole, constant numbers were obtained, suggesting that substitution of co-trimoxazole by ciprofloxacin for treatment of urinary tract infections contributed to the spread of $C$. difficile [13]. However, prescription rates of ciprofloxacin exhibited only subordinate seasonal dependences in most European countries [14] well as in our region [13] and can not explain seasonal fluctuations of CDI between 2006 and 2009.

The most frequently found PCR ribotypes, $C$. difficile 001 and 027 exhibited resistance to moxifloxacin and erythromycin confirming previous results $[3,11$, $15,16]$ while the less common PCR ribotypes predominantly showed low MICs.

These antibiotics are usually prescribed for treatment of respiratory tract infections. Since regional prescription numbers of these antibiotics also showed seasonal fluctuations paralleling Tcd findings [9], we hypothesized that antibiotic treatment of respiratory infections may trigger the development of CDI in patients previously colonized with resistant $C$. difficile. This observation suggests the existence of an association between fluoroquinolone and macrolideresistant $C$. difficile and antimicrobial consumption. However, between 2000 and 2006 in our region prescription numbers of moxifloxacin and macrolide antibiotics correlated only weakly to the number of CDI [12]. Unfortunately, the former study comprised only one year (2006) of the period with marked seasonal fluctuations (2006-2009) explaining the low association of the consumption of 'respiratory' antibiotics. Further studies may be helpful to further explore this association.

Since most respiratory infections are caused by viruses it could be argued that restriction of antibiotic use for treatment of respiratory infections may result in decreasing CDI numbers. CDI usually affects elderly patients [3] and elderly individuals are also more susceptible for severe pneumococcal disease. Consequently, delay of pneumonia treatment might result in a fatal outcome making it doubtful to recommend a general waiving of antibiotic treatment.

In a recent study performed in the USA on the association of seasonal $C$. difficile with influenza, it was shown that antibiotic treatment of respiratory infections induced development of CDI [17]. In a following study the authors calculated that a reduction of influenza activity by $20 \%$ would reduce prescription numbers of 'respiratory' fluoroquinolones by $8 \%$ [18]. Therefore, an increase of influenza vaccination coverage might also result in a lower number of CDI.

A seasonal distribution of CDI incidence was also described in other recent studies from Northern America (Canada) and Europe (Wales) [19, 20]. In these studies, the number of patients with CDI was higher in the winter months, even after correction for total number of patients at risk for in-hospital CDI. In our study, we took the number of patients tested for CDI into account, which did not show a distinct seasonal distribution until 2007. Therefore, we concluded that CDI tests are requested in patients with a similar degree of suspicion in winter months (percentage of positive results are similar per season), but there are more patients under suspicion during winter.

A limitation of the present study is the lack of clinical data. Furthermore, we were only able to differentiate between hospital and community onset of CDI. In the past years community-acquired CDI have become a focus of attention. In a recent study [21] $44 \%$ of CDI cases had a community onset suggesting a spreading of CDI from hospitals to the community. In the present study, the inclusion of data from inpatients and outpatients allows a more comprehensive analysis of the regional situation than restricting the analyses to hospital demission data [2, 17, 19] or on data reported to the health authorities. In 2009 a total of 67 cases of CDI with severe courses and three C. difficile 027 had been notified in Bavaria (personal communication, Dr Bettina Weiß, RKI). Although our region (governorate Oberpfalz) represents less than $10 \%$ of the Bavarian inhabitants we identified ninefold more $C$. difficile 027 than reported for the whole of Bavaria (27 vs. 3). Therefore, RKI criteria for CDI notifications are not sufficient to obtain representative data to understand the epidemiology of C. difficile and the impact of type 027 on CDI will be strongly underestimated when only data reported to the health authorities are used.

Diarrhoea caused by norovirus also exhibits distinct seasonality with increased activity in the winter months. Simultaneous $C$. difficile and norovirus infections have been described previously [22, 23]. From 2007 to 2009 the number of notified norovirus infections in our region coincided with increased numbers of CDI. However, in a detailed analysis $C$. difficile was 
not associated with outbreaks of viral gastroenteritis [24]. Nevertheless, this coincidence indicates that testing for CDI and for norovirus should belong to a panel of tests for agents of nosocomial diarrhoea in winter months.

In the present study $C$. difficile 027 was isolated from stool samples of out- and inpatients while this strain had not been observed in a previous regional study [3]. Strain 027 was not isolated from German inpatients in a recent study [25]. In contrast to these reports, $C$. difficile 027 has been isolated from 24 German patients and it was discussed that an outbreak scenario contributed to the spread of this strain [8]. In the present study, however, strain 027 was found in 18 hospitals and no outbreaks were reported indicating that this strain is endemic in our region.

Ten isolates belonged to ribotype 078 previously isolated exclusively from hospitalized patients suggesting nosocomial spread. In this study, the isolation rate of this ribotype was low, coinciding with results provided by previous studies [3, 7, 25]. Interestingly, this strain was initially prevalent in farm animals in the USA and The Netherlands [26, 27]. However, it has not yet been examined if $C$. difficile 078 is prevalent in livestock in our region. Surprisingly, two isolates belonged to ribotype 106. While this ribotype was commonly found in Great Britain and Ireland it was not prevalent in other European countries [25].

In summary we show that in a rural region in Southeastern Germany the number of CDI markedly increased during recent years and that a distinct seasonal association with peaks in winter months has been apparent since 2006. Resistance to 'respiratory' antibiotics seem to be a probable reason of increased C. difficile numbers in winter months.

\section{ACKNOWLEDGEMENTS}

The authors thank Bettina Weiß for providing the number of notified infections caused by $C$. difficile 027 in 2009 .

\section{DECLARATION OF INTEREST}

None.

\section{REFERENCES}

1. Barbut F, Jones G, Eckert C. Epidemiology and control of Clostridium difficile infections in healthcare settings: an update. Current Opinon in Infectious Diseases 2011; 24: $370-376$.
2. Vonberg RP, Schwab F, Gastmeier P. Clostridium difficile in discharged inpatients, Germany. Emerging Infectious Diseases 2007; 13: 179-180.

3. Borgmann S, et al. Increased number of Clostridium difficile infections and prevalence of Clostridium difficile PCR ribotype 001 in southern Germany. Eurosurveillance $2008 ; \mathbf{1 3}$ : pii $=19057$.

4. Goorhuis A, et al. Emergence of Clostridium difficile infection due to a new hypervirulent strain, polymerase chain reaction ribotype 078. Clinical Infectious Diseases 2008; 47: 1162-1170.

5. Robert Koch Institute. Clostridium difficile infections. Notifications of infections according to the IFSG (infection prevention law) between 2008 and 2009 [in German]. Epidemiologisches Bulletin 2010; 10: 87-89.

6. Kleinkauf $\mathbf{N}$, et al. Confirmed cases and report of clusters of severe infections due to Clostridium difficile PCR ribotype 027 in Germany. Eurosurveillance 2007; 12: pii $=3307$.

7. Zaiss NH, Witte W, Nübel U. Fluoroquinolone resistance and Clostridium difficile, Germany. Emerging Infectious Diseases 2010; 16: 675-677.

8. Arvand M, et al. Clostridium difficile ribotypes 001, 017, and 027 are associated with lethal C. difficile infection in Hesse, Germany. Eurosurveillance 2009; 14: pii $=19403$.

9. Borgmann S, et al. Prescriptions of broad-spectrum antibiotics to outpatients do not match increased prevalence and antibiotic resistance of respiratory pathogens in Bavaria. Polish Journal of Microbiology 2009; 58: 105-110.

10. Bidet $\mathbf{P}$, et al. Development of a new PCR-ribotyping method for Clostridium difficile based on ribosomal RNA gene sequencing. FEMS Microbiology Letters 1999; 175: 261-266.

11. Barbut F, et al. Prospective study of Clostridium difficile infections in Europe with phenotypic and genotypic characterization of the isolates. Clinical Microbiology and Infection 2007; 13: 1048-1057.

12. Borgmann S, et al. Association of ciprofloxacin prescriptions to outpatients to Clostridium difficile infections. Eurosurveillance 2010; 15: pii $=19479$.

13. Borgmann S, et al. Ciprofloxacin treatment of urinary infections results in increased resistance of urinary E. coli to ciprofloxacin and co-trimoxazole. Polish Journal of Microbiology 2009; 58: 371-373.

14. Goossens H, et al. ESAC Project Group. Outpatient antibiotic use in Europe and association with resistance: a cross-national database study. Lancet 2005; 365: 579-587.

15. Brazier JS, et al. Distribution and antimicrobial susceptibility patterns of Clostridium difficile PCR ribotypes in English hospitals, 2007-08. Eurosurveillance 2008; 13: pii $=19000$.

16. McDonald LC, et al. An epidemic, toxin gene-variant strain of Clostridium difficile. New England Journal of Medicine 2005; 353: 2433-2441.

17. Polgreen PM, et al. A time-series analysis of Clostridium difficile and its seasonal association with influenza. Infection Control and Hospital Epidemiology 2010; 31 : 382-387. 
18. Polgreen PM, et al. Respiratory fluoroquinolone use and influenza. Infection Control and Hospital Epidemiology 2011; 32: 706-709.

19. Gilca R, et al. Epidemiological patterns and hospital characteristics associated with increased incidence of Clostridium difficile infection in Quebec, Canada, 1998-2006. Infection Control and Hospital Epidemiology 2010; 31 : 939-934.

20. National Health Service for Wales. All Wales $C$. difficile surveillance report commentary October 2009 to September 2010 (http://www.wales.nhs.uk/sites3/page. cfm?orgid $=379 \&$ pid $=18490)$. Accessed 21 February 2011.

21. Kuntz JL, et al. Incidence of and risk factors for community-associated Clostridium difficile infection: A nested case-control study. BMC Infectious Diseases 2011; 11: 194 .

22. Bignardi GE, Staples K, Majmudar N. A case of norovirus and Clostridium difficile infection: casual or causal relationship? Journal of Hospital Infection 2007; 67: 198-200.

23. Lukkarinen $\mathbf{H}$, et al. Clostridium difficile ribotype 027 -associated disease in children with norovirus infection. Pediatric Infectious Disease Journal 2009; 28: 847-848.

24. Svraka S, et al. Clostridium difficile is not associated with outbreaks of viral gastroenteritis in the elderly in the Netherlands. European Journal of Clinical Microbiology and Infectious Diseases 2010; 29: 677-682.

25. Bauer MP, et al. Clostridium difficile infection in Europe: a hospital-based survey. Lancet 2011; 377: 63-73.

26. Goorhuis A, et al. Clostridium difficile PCR ribotype 078: an emerging strain in humans and in pigs? Journal of Clinical Microbiology 2008; 46: 1157.

27. Keel K, et al. Prevalence of PCR ribotypes among Clostridium difficile isolates from pigs, calves, and other species. Journal of Clinical Microbiology 2007; 45: 1963-1964. 